\title{
Recent advances in pulse oximetry
} Maxime Cannesson ${ }^{1}$ and Pekka Talke ${ }^{2 *}$

\author{
Addresses: ${ }^{1}$ Department of Anesthesiology and Perioperative Care, University of California, Irvine, California 92868, USA; ${ }^{2}$ Department of \\ Anesthesia and Perioperative Medicine, University of California, San Francisco, California 94143, USA \\ *Corresponding author: Pekka Talke (talkep@anesthesia.ucsf.edu) \\ FI000 Medicine Reports 2009, I:66 (doi:I0.34I0/MI-66)
}

The electronic version of this article is the complete one and can be found at: http://FI000.com/Reports/Medicine/content/I/66

\begin{abstract}
Conventional pulse oximetry uses two wavelengths of light (red and infrared) transmitted through a finger and a photodetector to analyze arterial hemoglobin oxygen saturation and pulse rate. Recent advances in pulse oximetry include: extended analysis of the photo plethysmographic waveform; use of multiple wavelengths of light to quantify methemoglobin, carboxyhemoglobin and total hemoglobin content in blood; and use of electronic processes to improve pulse oximeter signal processing during conditions of low signal-to-noise ratio. These advances have opened new clinical applications for pulse oximeters that will have an impact on patient monitoring and management.
\end{abstract}

\section{Introduction and context}

Pulse oximeters are used daily for arterial hemoglobin oxygen saturation $\left(\mathrm{SpO}_{2}\right)$ and pulse rate $(\mathrm{PR})$ monitoring in various clinical settings. Conventional pulse oximetry uses two wavelengths of light (red and infrared) transmitted through the distal phalanx of the finger. This transmitted light is sensed by a photodetector. In simplistic terms, pulse oximeters use the red-to-infrared signal ratio and proprietary calibration tables to calculate $\mathrm{SpO}_{2}$. The photo plethysmographic waveform (infrared) is used to determine PR. Under normal conditions basic pulse oximeter technology functions extremely well. However, under more challenging conditions, such as poor signal-to-noise ratio, during movement artifacts, or during significant hypoxic episodes, the older technologies have limitations. Over the past several years pulse oximeter technology has advanced significantly as a result of attempts to improve $\mathrm{SpO}_{2}$ and $\mathrm{PR}$ analysis during these challenging conditions.

Recently, pulse oximeter technology has also expanded from measuring $\mathrm{SpO}_{2}$ and $\mathrm{PR}$ to measuring other hemoglobin species (carboxyhemoglobin, methemoglobin, total hemoglobin) using multiwavelength analysis, and the technology has expanded morphological analysis of the photo plethysmographic waveform (respiratory variations, perfusion index, venous pulse). We will discuss these recent advances in pulse oximetry in more detail.

\section{Recent advances}

Morphological analysis of the photo plethysmographic waveform

Despite its simple appearance, the pulse oximeter photo plethysmographic waveform is a highly complex signal that contains far more information than just PR. The photo plethysmograph waveform is based on a signal proportional to infrared light absorption between an emitter and a photo detector, which are usually placed on the opposite sides of the fingertip (transmission photo plethysmography). The original raw signal is the current generated by the photo detector. In most pulse oximeters this signal undergoes some processing before being displayed on the device. The raw plethysmograph waveform has two components, frequently referred to as $\mathrm{DC}$ and AC. The DC component is the nonpulsatile component of the plethysmograph signal and is inversely proportional to light absorption and scattering by nonpulsatile tissues of the fingertip, including nonpulsatile blood (arterial and venous). The AC component is the pulsatile component of the plethysmograph waveform. An assumption is made that the AC component represents blood in the arterial side of the circulation. 
Photo plethysmography measures changes in volume of the finger. The larger the blood volume in the finger (vasodilation), the more light is being absorbed by the finger. Thus, less light passes through the finger and the current generated by the photodetector is smaller. So, during systole the amount of light transmitted through the finger is less than during diastole, and the original plethysmograph signal resembles a mirror image of an arterial blood pressure waveform. To make it easier for the clinicians to interpret the plethysmograph waveform, most devices invert the image on their display. In addition, the plethysmograph waveform is frequently auto-scaled to fit the display area. As a result, potential physiologic information that may be in the DC and AC components of the signal is lost (not utilized).

It is clear that the plethysmograph waveform contains important physiological information. Changes in the AC and DC components of the plethysmograph waveform have been related to vasomotor tone [1-5]. The DC component is also influenced by respiration and may contain information relating to patients' fluid status [6-9]. Recent advances in pulse oximetry have focused on the morphological analysis of the plethysmographic waveform.

One variable that is derived from the plethysmograph waveform is perfusion index (PI). PI is defined as AC/ DC $\times 100 \%$ of the plethysmograph waveform, and is now displayed on some pulse oximeters. In general terms, PI reflects peripheral vasomotor tone. Low PI suggests peripheral vasoconstriction (or severe hypovolemia) and high PI suggests vasodilation. PI is sensitive to several things such as temperature of the finger, exogenous vasoactive drugs, sympathetic nervous system tone (pain, anxiety, and so on) and stroke volume.

Another variable that is derived from the plethysmograph waveform is pleth variability index (PVI). PVI is a relatively new parameter that is provided currently by one pulse oximeter manufacturer. PVI quantifies the variability in plethysmograph waveform due to respiration and is thought to be a surrogate measure of intravascular volume. It is defined as ( $\mathrm{PI}$ max - PI $\min$ )/ $\mathrm{PI}_{\max } \times 100 \%$. Several similar parameters (pulse pressure variation, delta up/delta down, systolic blood pressure variability) have been previously derived from the arterial blood pressure waveform.

Use of pulse oximeters for monitoring patients' intravascular volume status

Dynamic indicators of fluid responsiveness (including the respiratory variations in the pulse oximeter plethysmographic waveform amplitude) rely on cardiopulmonary interactions in mechanically ventilated patients [10]. Briefly, during lung inflation, the increase in intrathoracic pressure induces a decrease in right ventricular venous return. This phenomenon leads to a decrease in right ventricular stroke volume that is transmitted to the left ventricle. The inverse phenomenon is observed during exsufflation. These phenomena are responsible for respiratory variations in the left ventricular stroke volume and can be detected as variations in systemic arterial pulse pressure [10]. Shamir et al. [11] were the first to describe the respiratory variations in the plethysmographic waveform in patients presenting with various degrees of hypovolemia. In 2005, the respiratory variation in the pulse oximeter waveform amplitude $(\triangle \mathrm{POP})$ was described. $\triangle \mathrm{POP}$ was then tested in clinical settings and was shown to be sensitive to venous return in mechanically ventilated patients [12] and to be an accurate predictor of fluid responsiveness [6]. Predicting fluid responsiveness with a noninvasive device afforded a novel approach to hemodynamic management that was previously only available with the use of invasive monitoring [13]. PVI, as described above, is a clinically available continuous measurement of the respiratory variations of the pulse oximeter plethysmographic waveform amplitude. Cannesson et al. [14] reported that a PVI value $>14 \%$ predicted fluid responsiveness. Future studies will define the utility of PVI to guide intravascular volume management.

\section{Use of pulse oximeters for regional anesthesia}

Local anesthetic-induced sympathectomy during regional anesthesia causes peripheral vasodilation. This vasodilation can be quantified using the plethysmograph waveform (PI). Thus, PI has been evaluated as a predictor of the success of regional anesthesia. Two recent studies have used PI to monitor intravascular epinephrineinduced changes in vasomotor tone in patients receiving epidural anesthesia during general anesthesia [4,5]. These studies have shown that PI was a good indicator of intravascular epinephrine injection under general anesthesia (intravascular epinephrine injection induced a significant decrease in PI).

\section{Other morphological analysis}

Morphological analysis of the plethysmographic waveform can provide information regarding venous pulsation or analgesia (analysis of the slope of the plethysmographic waveform ascending portion). These indices are still under investigation. A forehead pulse oximeter sensor can be interfered with by pulsation of venous blood in the forehead. This would result in falsely low $\mathrm{SpO}_{2}$ readings. To eliminate venous blood pulsations under the forehead pulse oximeter probe, an 
elastic tensioning headband is used to compress the veins.

One pulse oximeter manufacturer has explored the use of photo plethysmographic waveform amplitude and PR to estimate the nociception-antinociception balance of the anesthetized patient. This surgical pleth index (not commercially available; previously surgical stress index) reflects sympathetic nervous system activation and potentially insufficient analgesia. The use of surgical pleth index to guide use of intra-operative analgesics will be studied in the future.

\section{Multiwavelength analysis \\ Carboxyhemoglobin and methemoglobin measurements}

Common pulse oximeters measure tissue light transmission at two wavelengths to determine arterial oxygen saturation as the ratio of oxyhemoglobin to total hemoglobin. This assumption hypothesized that only two components absorb light in the blood: oxyhemoglobin and reduced hemoglobin. However, other hemoglobin species such as methemoglobin, carboxyhemoglobin, and other dyshemoglobins also absorb light in the blood. A recently developed pulse oximeter platform uses multiple wavelengths of light to analyze several different hemoglobins. Using eight or more wavelengths of light, these devices can measure carboxyhemoglobin and methemoglobin concentrations in humans noninvasively and continuously [15].

\section{Total hemoglobin measurements}

In 2009 another innovation in multiwavelength pulse oximetry was released. Using 12 or more wavelengths of light, this pulse oximeter allows for continuous, noninvasive total hemoglobin monitoring. Future studies will evaluate the clinical utility of this device.

\section{Alternative analysis, alternative technologies \\ New generation pulse oximeters: motion artifacts, low perfusion and low arterial oxygen saturation reading}

Motion, low perfusion, and optical shunting can induce considerable errors into pulse oximeter accuracy, resulting in loss of data, inaccurate readings, and false alarms [16]. Most clinicians would agree that motion artifacts and the resulting false alarms have been the most significant drawback to using pulse oximetry in the critical care setting. Motion artifacts result in a low signalto-noise ratio, with $\mathrm{SpO}_{2}$ being driven to lower than actual readings due to venous motion. This venous component is exacerbated by low perfusion. Moreover, all pulse oximeters have been empirically calibrated by desaturating healthy volunteers in an oxyhemoglobin range of $100 \%$ to $70 \%$ [16]. The resulting sensor calibration curves reside within most monitors. Some sensors include small digital memory chips that contain sensor-specific calibration curves.

To be a useful clinical tool, pulse oximeters should give real-time, continuous, and accurate measurements over a wide range of arterial oxygen saturation values, during all types of patient motion, continuous and intermittent, aperiodic and rhythmic, and during low perfusion. There appear to be significant differences in the abilities of the currently available motion-tolerant technologies to achieve these goals, but all motion-tolerant technologies appear to perform better than conventional technologies [17]. Although more advances will be made in these areas, it is likely that the improvements will be relatively small due to the excellence of currently available measure-through-motion-and-low-perfusion technology.

\section{Implications for clinical practice}

Recent advances in pulse oximeters have brought these devices into new and important clinical situations. They have evolved from relatively simple arterial oxygen saturation monitors to technologically advanced instruments that are capable of measuring several hemoglobin species and physiologic parameters. Today, pulse oximeters have the ability to be used for hemodynamic management, regional anesthesia, hemoglobin monitoring, and carboxyhemoglobin and methemoglobin detection. The new generation of pulse oximeters can provide accurate measurements in challenging situations such as low perfusion, the presence of motion artifacts, and low arterial oxygen saturation. The photo plethysmographic waveform is used to analyze new parameters that may have significant impacts on future clinical practice and, undoubtedly, new plethysmograph-derived parameters and light transmission-related technologies will be developed. Clinical research will be needed to define the utility of these technologies, and to identify new monitoring opportunities.

\section{Abbreviations}

AC, pulsatile component of the plethysmograph signal; $\mathrm{DC}$, nonpulsatile component of the plethysmograph signal; $\triangle \mathrm{POP}$, respiratory variations in the pulse oximeter waveform amplitude; PI, perfusion index; PR, pulse rate; PVI, pleth variability index; SPI, surgical pleth index; $\mathrm{SpO}_{2}$, arterial oxygen saturation.

\section{Competing interests}

MC is a consultant for Masimo (Irvine, CA) and for Covidien (Mansfield, MA). PT is a consultant for Masimo. 


\section{References}

I. Shelley KH, Murray WB, Chang D: Arterial-pulse oximetry loops: a new method of monitoring vascular tone. J Clin Monit 1997, 13:223-8.

2. Landsverk SA, Hoiseth LO, Kvandal P, Hisdal J, Skare O, Kirkeboen KA: Poor agreement between respiratory variations in pulse oximetry photoplethysmographic waveform amplitude and pulse pressure in intensive care unit patients. Anesthesiology 2008, 109:849-55.

3. Hummler HD, Engelmann A, Pohlandt F, Högel J, Franz AR: Decreased accuracy of pulse oximetry measurements during low perfusion caused by sepsis: Is the perfusion index of any value? Intensive Care Med 2006, 32:|428-31.

4. Mowafi HA, Ismail SA, Shafi MA, Al-Ghandi AA: The efficacy of perfusion index as an indicator for intravascular injection of epinephrine-containing epidural test dose in propofolanesthetized adults. Anesth Analg 2009, 108:549-53.

Changes Clinical Practice

FI000 Factor 3.0 Recommended

Evaluated by Pekka Talke 03 Mar 2009

5. Mowafi HA, Arab SA, Ismail SA, Al-Ghamdi AA, Al-Metwalli RR: Plethysmographic pulse wave amplitude is an effective indicator for intravascular injection of epinephrine-containing epdirual test in sevoflurane-anesthetized pediatric patients. Anesth Analg 2008, 2008:|536-4I.

FI000 Factor 3.0 Recommended

Evaluated by Francis Veyckemans 04 Aug 2008

6. Cannesson M, Attof Y, Rosamel P, Desebbe O, Joseph P, Metton O, Bastien $O$, Lehot J]: Respiratory variations in pulse oximetry plethysmographic waveform amplitude to predict fluid responsiveness in the operating room. Anesthesiology 2007, 106: | 105-11.

7. Feissel M, Teboul JL, Merlani P, Badie J, Faller JP, Bendjelid K: Plethysmographic dynamic indices predict fluid responsiveness in septic ventilated patients. Intensive Care Med 2007, 33:993-9.
8. Shelley $\mathrm{KH}$ : Photoplethysmography: beyond the calculation of arterial oxygen saturation and heart rate. Anesth Analg 2007, 105:S31-6.

9. Shelley $\mathrm{KH}$, Jablonka DH, Awad AA, Stout RG, Rezkanna H, Silverman DG: What is the best site for measuring the effect of ventilation on the pulse oximeter waveform? Anesth Analg 2006, 103:372-7.

10. Michard $\mathrm{F}$ : Changes in arterial pressure during mechanical ventilation. Anesthesiology 2005, 103:419-28; quiz 449-5

II. Shamir M, Eidelman LA, Floman Y, Kaplan L, Pizov R: Pulse oximetry plethysmographic waveform during changes in blood volume. Br J Anaesth 1999, 82:178-81.

12. Cannesson M, Besnard C, Durand PG, Bohe J, Jacques D: Relation between respiratory variations in pulse oximetry plethysmographic waveform amplitude and arterial pulse pressure in ventilated patients. Crit Care 2005, 9:R562-8.

13. Desebbe O, Cannesson M: Using ventilation induced plethysmographic variations to optimize patient fluid status. Curr Opin Anaesthesiol 2008, 2 I:772-8.

FI000 Factor 6.0 Must Read Evaluated by Marc De Kock 09 Jan 2009

14. Cannesson M, Desebbe O, Rosamel P, Delannoy B, Robin J, Bastien O, Lehot J): Pleth variability index to monitor the respiratory variations in the pulse oximeter plethysmographic waveform amplitude and predict fluid responsiveness in the operating theatre. Br J Anaesth 2008, I0 I:200-6.

FI000 Factor 3.0 Recommended Evaluated by Paul Barash 04 Aug 2008

15. Barker SJ, Curry J, Redford D, Morgan S: Measurements of carboxyhemoglobin and methemoglobin by pulse oximetry: a human volunteer study. Anesthesiology 2007, 105:892-7.

16. Petterson MT, Begnoche VL, Graybeal JM: The effect of motion on pulse oximetry and its clinical significance. Anesth Analg 2007, I 05(6 Suppl):S78-84.

17. Aoyagi T, Fuse M, Kobayashi N, Machida K, Miyasaka K: Multiwavelength pulse oximetry: theory for the future. Anesth Analg 2007, I 05(6 Suppl):S53-8. 